\title{
Wearable, low-cost device for monitoring cerebral blood flow with speckle contrast optical spectroscopy
}

\author{
Manish Verma $^{1, *}$, Tanja Dragojević ${ }^{1}$, Ameer Ghouse ${ }^{1}$, Laura Moreno Carbonell ${ }^{1}$, Umut Karadeniz ${ }^{1}$, Daniel \\ Fernandez Cuenca ${ }^{1}$, Ernesto E. Vidal-Rosas ${ }^{1}$, Davide Tamborini ${ }^{2}$, Davide Portaluppi ${ }^{2}$, Mauro Buttafava ${ }^{2}$, Federica \\ Villa ${ }^{2}$ and Turgut Durduran ${ }^{1,3}$ \\ ${ }^{1}$ ICFO-The Institute of Photonic Sciences, Barcelona Institute of Science and Technology, Spain, ${ }^{2}$ Politecnico di Milano, Dipartimento di Elettronica, \\ Informatione e Bioingegneria, Milan, Italy, ${ }^{3}$ Institució Catalana de Recerca i Estudis Avançats (ICREA), Spain; \\ *mv4manishverma@gmail.com
}

\begin{abstract}
A wearable, low-cost device was developed for measuring cerebral blood flow using speckle contrast optical spectroscopy. We present its design and results from different protocols during realistic scenarios taking advantage of it being a wearable device. (C) 2020 The Author(s)

OCIS codes: $170.1470,170.2655,110.6150,170.3880$
\end{abstract}

\section{Introduction:}

Cerebral blood flow (CBF) is an important biomarker of health, development and pathology. Its practical, non-invasive measurement has applications in numerous areas [1-4]. In recent years, there have been a lot of advancements in noninvasive measurement of CBF through diffuse optical techniques [5] namely, diffuse correlation spectroscopy (DCS) [6] and speckle contrast optical spectroscopy (SCOS) [7]. These techniques utilize near infrared light to access spectroscopic and transport properties of deep cerebral tissues. In DCS, the temporal fluctuations from light collected from a single mode fiber is directly measured in the form of the intensity auto-correlation function. There are various restrictions placed on DCS hardware which make its current implementations costly and bulky. On the other hand, in SCOS, the speckle contrast is utilized as a data-type that allows for improved scalability and cost reduction on the detection side. Recently, we have introduced a custom single photon avalanche diode (SPAD) array with associated micro-electronics that enabled us to demonstrate the feasibility of neuro-monitoring with this compact, low-cost detector with SCOS [8]. In this work, we demonstrate a complete wearable system that suitable for use in realistic scenarios such as walking, social interactions and sports. We describe the wireless, battery operated device, the head-gear and characterize it on in vivo tests. Finally, we demonstrate its utility in various tasks.

\section{Device:}

The device, shown in Fig.1(a), consist of a custom designed 32x2 SPAD array detector [8] with a new $90^{\circ}$ objective lens, a compact, in-house designed single-mode laser diode and its driver and fiber optical coupling for light delivery. The laser, the control and safety electronics, the battery and a single-board computer (Raspberry Pi Foundation, UK). The size and the weight is suitable to be carried in as a backpack. The detector communication and power is supplied by a standard USB connection. The device is capable of independent operation on battery for more than three hours. The single-board computer creates its own wireless network and all the control, real-time processing and graphical interface is handled by a custom written software. The user interacts with the system by connecting to the wireless network from a handheld device, e.g. a smart-phone or a tablet, using a remote desktop software. All data is stored internally and can be downloaded through Wi-Fi interface or by using a USB connection. The system is designed to be versatile with various automated safety mechanisms for example for using a touch sensor to controlling the laser, provides different wired and wireless inputs/outputs for synchronization and marking.

As previously described, the embedded microelectronics of the SPAD array calculates the noise corrected, multiexposure speckle contrast data for each pixel [8]. The on-board software then fits this data in real-time using the correlation diffusion model for the speckle contrast. By using appropriate approximations, we obtain the absolute value of the blood-flow index every two seconds.

\section{Results:}

Figure 1(b) shows an arm-cuff occlusion experiment where the probe is placed on the human forearm whose results are displayed in real-time (Figure 1 (c)). Figure 1(c) shows the raw speckle contrast $\left(\mathrm{K}^{2}\right)$ over-time for a chosen exposure time (bottom row) as well as the relative changes in blood flow according to a baseline period that was selected by the user. We are able to observe the well-known features of the blood flow changes due to arterial cuff occlusion. Figure 1(d) shows the use of the system during interactive video gaming. We will demonstrate different results during mobile tasks such as walking through obstacles and exercising (Figure 1(e) and Figure 1(f) respectively), social interactions and others. Overall, 
this prototype system paves the way for the next generation neuro-monitors using cerebral blood flow as the biomarker. We will describe its extensions to multiple channels and further reductions in footprint and weight.

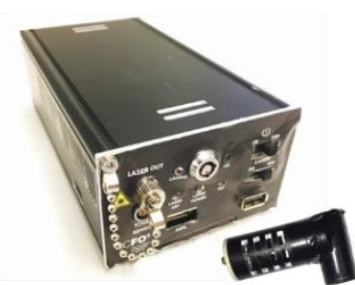

(a)

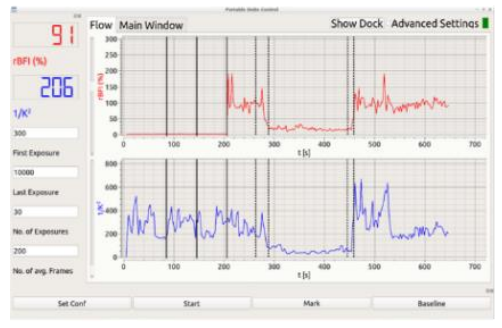

(c)

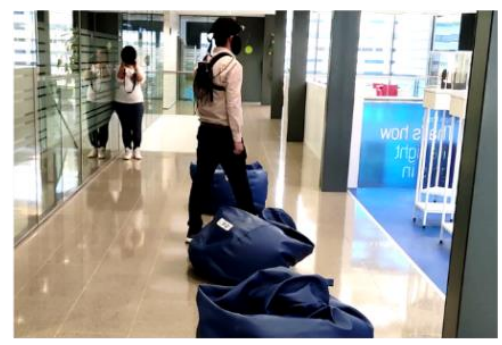

(e)

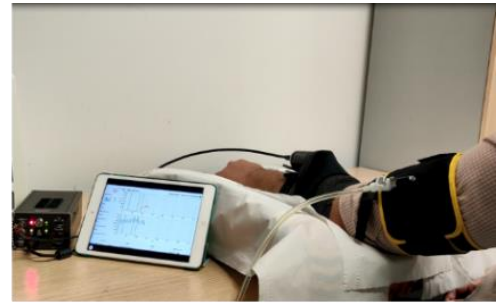

(b)

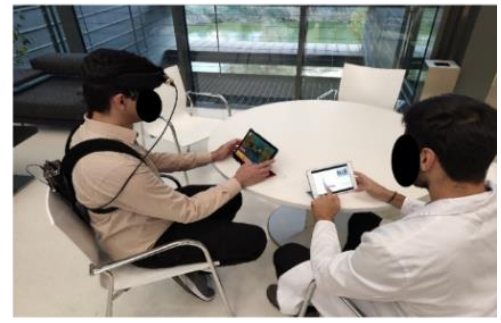

(d)

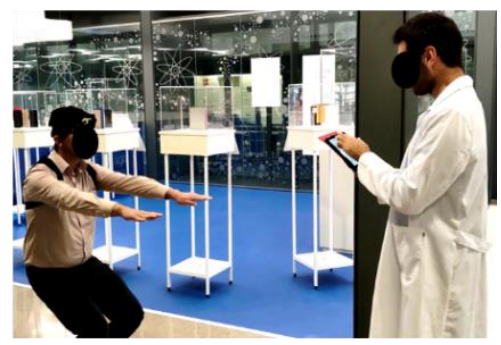

(f)

Figure 1: (a) The portable SCOS device and Probe. (b) An arterial cuff occlusion experiment on human forearm with the device. (c) screen-shot of the real-time blood flow data of the arm-cuff occlusion experiment. (d), (e) and (f) show real-time monitoring of $\mathrm{CBF}$ while playing an interactive video game on a tablet, walking through obstacles and during exercising respectively.

Acknowledgment: The project was funded by Fundacio CELLEX Barcelona, Ministerio de Econonia y Competividad FEDER (PHOTODEMENTIA, DPI2015-64358-C2-1-R), Instituto de Salud Carlos III/FEDER (MEDPHOTAGE, TS16/00087), the "Severo Ochoa" Programme for Centres of Excellence in R\&D (SEV-2015-0522) and the Obra social "la Caixa" Foundation (LlumMedBcn), Fundacio "La Marato TV3".

\section{References:}

1. M. HONDA et. al, "Early Cerebral Circulation Disturbance in Patients Suffering from Severe Traumatic Brain Injury (TBI): A Xenon CT and Perfusion CT Study" Neurol. Med. Chir. (Tokyo). 56(8) 501-509 (2016).

2. D. W. Marion et al., "Acute regional cerebral blood flow changes caused by severe head injuries" Journal of Neurosurgery 74 (3) (1991).

3. E. Rostami et al., "Imaging of Cerebral Blood Flow in Patients with Severe Traumatic Brain Injury in the Neurointensive Care" Front Neurol. 5 (114) (2014);

4. K. Kisler et al., "Cerebral blood flow regulation and neurovascular dysfunction in Alzheimer disease" Nature Reviews Neuroscience, 8, 419434 (2017).

5. T. Durduran et al. "Diffuse optics for tissue monitoring and tomography" Rep. Prog. Phys. 73, 076701 (43pp) (2010).

6. T. Durduran et al., "Diffuse correlation spectroscopy for non-invasive, micro-vascular cerebral blood flow measurement" NeuroImage 85, 5163 (2014).

7. C. P. Valdes et al., "Speckle contrast optical spectroscopy, a non-invasive, diffuse optical method for measuring microvascular blood flow in tissue" Biomedical Optics Express, 5 (8), 2769-2784 (2014).

8. T. Dragojević et al, "Compact, multi-exposure speckle contrast optical spectroscopy (SCOS) device for measuring deep tissue blood flow" Biomedical Optics Express, 9 (1), 322-334 (2018). 